\title{
The upstream boundary condition influences the leaflet opening dynamics in the numerical FSI simulation of an aortic BMHV
}

\author{
Sebastiaan Annerel ${ }^{1, *, \uparrow}$, Joris Degroote ${ }^{1}$, Tom Claessens ${ }^{2}$, Patrick Segers ${ }^{3}$, Pascal \\ Verdonck $^{3}$ and Jan Vierendeels ${ }^{1}$ \\ ${ }^{1}$ Department of Flow, Heat and Combustion Mechanics, Ghent University, \\ Sint-Pietersnieuwstraat 41, B-9000 Ghent, Belgium \\ ${ }^{2}$ BioMech, Department of Mechanics, University College Ghent, \\ Valentin Vaerwykweg 1, B-9000 Ghent, Belgium \\ ${ }^{3}$ IBiTech-bioMMeda, Ghent University, De Pintelaan 185, B-9000 Ghent, Belgium
}

\begin{abstract}
SUMMARY
In this paper, the influence of the upstream boundary condition in the numerical simulation of an aortic bileaflet mechanical heart valve (BMHV) is studied. Three 3D cases with different upstream boundary conditions are compared. A first case consists of a rigid straight tube with a velocity profile at its inlet. In the second case, the upstream geometry is a contracting left ventricle (LV), positioned symmetrically with respect to the valve. In the last case, the LV is positioned asymmetrical with respect to the valve. The cases are used to simulate the same 3D BMHV. The change in time of the LV volume is calculated such that the flow rate through the valve is identical in each case. The opening dynamics of the BMHV are modelled using fluidstructure interaction (FSI). The simulations show that differences occur in the leaflet movement of the three cases. In particular, with the asymmetric LV, one of the leaflets impacts the blocking mechanism at its open position with a $34 \%$ higher velocity than when using the velocity profile, and with an $88 \%$ higher velocity than in the symmetric LV case. Therefore, when simulating such an impact, the upstream boundary condition needs to be chosen carefully.
\end{abstract}

Submitted 30 May 2011;

KEY WORDS: Boundary condition; bileaflet mechanical heart valve; left ventricle; FSI

\footnotetext{
* Correspondence to: Sebastiaan Annerel, Department of Flow, Heat and Combustion Mechanics, Ghent University, Sint-Pietersnieuwstraat 41, B-9000 Ghent, Belgium

$\dagger$ E-mail: Sebastiaan.Annerel@UGent.be

Contract/grant sponsor: Special Research Fund of Ghent University Association (BOF)

Contract/grant sponsor: Research Foundation—Flanders (FWO)
} 


\section{INTRODUCTION}

The dynamics of a bileaflet mechanical heart valve (BMHV) depend on passive movement, i.e. the opening and closure of the leaflets are governed by the pressure gradients and flow fields in the heart and arteries (in case of atrioventricular valves) [1]. For the numerical simulation of BMHVs, several types of boundary conditions can be used. However, since the dynamic movement of the valve leaflets is interacting with the resulting flow field, the (geometrical) boundary conditions need to be chosen carefully. Commonly an unsteady inflow velocity profile is imposed at the upstream inlet. The shape of the velocity profile at the inlet can be chosen uniform $[2,3,4,5]$, trapezoidal $[6,7]$, plug flow $[8,9,10,11]$, or fully developed [12,13]. Another approach is to implement a boundary condition that incorporates the upstream geometry and dynamics of the blood flow. For a BMHV in the aortic position, this can be done by a model of the contracting left ventricle (LV). When the LV contracts, a flow into the aorta is generated, forcing the opening of the aortic valve. The filling of the LV is controlled by the mitral valve. In literature, models of the LV are used wherein the position of the aortic valve with respect to the mitral valve is taken coplanar $[14,15,16,17,18]$ or non-coplanar $[19,20,21,22,23,24]$.

In previous work it was shown that significant differences in the geometry downstream of the valve can induce significant differences in valve leaflet kinematics [3]. In this paper, the use of a velocity profile and a contracting LV as upstream boundary condition is discussed and evaluated through $3 \mathrm{D}$ numerical simulations.

Moreover, in many experimental tests [25-29], the upstream and downstream geometry is simplified into a tube with an imposed flow rate, whether with or without downstream (axi)symmetric Valsalva sinuses. Therefore, the comparison performed in this study between the different inflow profiles gives a first quantification of the error that is made in such experimental setups.

The paper is structured as follows. First, the details of the numerical simulations and the boundary conditions are discussed. Subsequently, the resulting valve leaflet kinematics, the dynamics and the flow fields are described and the spatial and temporal convergence (of the leaflet kinematics and the velocity fields) are investigated. Finally, the conclusions are discussed and summarized.

\section{METHODS}

In this section, the setup of the $3 \mathrm{D}$ cases is discussed. First, the numerical simulation of the dynamics of a BMHV is described. Subsequently, the details of the boundary conditions are discussed.

\section{$2.1 \quad$ Fluid-structure interaction simulation of the BMHV}

The numerical simulation of a BMHV is a complex fluid-structure interaction (FSI) problem because the movement of the leaflets strongly interacts with the surrounding fluid motion and, therefore, the dynamic equilibrium at the fluid-structure interface needs to be taken into account.

The dynamics of the BMHV with rigid leaflets is calculated by a recently developed FSI algorithm [3]. This strong coupling algorithm uses separated solvers for the flow and 
the structural domain. Its convergence process is accelerated by a dynamically changing relaxation matrix, as first developed in [2,20] and later optimised in [3]. The method is an extension of the one described in [30] and predicts the moments (and thus the angular accelerations) of the next coupling iteration through a linearization of Newton's Second Law with a finite difference approximation of the Jacobian. The components of this Jacobian are the derivatives of the moments (exerted by the flow on the leaflets) with respect to changes in leaflet angular accelerations and are numerically derived from the flow solver by variations of the leaflet positions. A detailed description of the FSI algorithm can be found in [3]. Moreover, in [2,3] it is shown that this (quasi-Newton) method outperforms more commonly used relaxation schemes (like the fixed relaxation and Aitken $\Delta^{2}$ relaxation) in needed coupling iterations per time step and CPU time.

The BMHV used in the simulations is a model of the $25 \mathrm{~mm}$ ATS Open Pivot Standard Heart Valve in aortic position with the orifice inner diameter measuring $20.8 \mathrm{~mm}[2,3]$. The valve model is simplified at the hinge regions for reasons of grid motion by cutting away the blocking mechanism and hinges at the casing, as can be seen in Figure 1. This model is previously used in $[2,3]$.

However, although the blocking mechanisms at the open and closed positions are cut away and are thus absent, their interaction with the leaflets is modelled by limiting the leaflet motion between a fully open and fully closed position, as described in $[2,3]$.

\subsection{Boundary Conditions}

Since the downstream conditions are not of interest in this study, the downstream geometry is simplified to a rigid straight tube with diameter $22 \mathrm{~mm}$. A physiological pressure is imposed at the outlet boundary, although in a rigid geometry the pressure level does not affect the flow field (since only the pressure gradient appears in the equations).

Upstream of the valve, the flow rate is specified. This is done by the use of different types of upstream boundary conditions, resulting in the three cases that are visualised in Figure 2.

In the first case, a rigid straight tube (with diameter $22 \mathrm{~mm}$ ) is placed upstream with a velocity profile imposed at the inlet (Figure 2(a)). The used velocity profile is an aortic flow pulse with a time cycle of $1 \mathrm{~s}$ and is displayed in Figure $3(a)$. It is the same uniform velocity profile as is used in $[2,3]$.

Secondly, an upstream (geometrically) contracting LV is used, as is done in [15]. Two cases are constructed. One case consists of a LV positioned symmetrically with respect to the rotation axes of the valve leaflets (Figure $2(b)$ ). In the other case, the LV is positioned asymmetrical with respect to the rotation axes of the valve leaflets (Figure 2(c)). Both models of the LV are simplified by assuming coplanarity of the mitral and aortic valve $[14,15,16,17,18]$. Since in this study only the opening of an aortic BMHV is analysed, solely the contraction of the LV needs to be simulated, with the mitral valve thus completely closed. Therefore, the assumption of coplanarity of the valves is sufficient. Moreover, the shape of the LV is modelled as a prolate spheroid, as is commonly done $[14,16,17,19,31,32,33,34]$. The long-to-short-axis ratio of the spheroid is kept constant at 2 throughout the contraction, which is considered the normal reference for a human LV [32]. 
The change in time of the LV volume is calculated such that the flow rate through the valve in the two LV cases is identical at every time level to the case with the velocity profile at the inlet. Therefore, an end-diastolic volume of $111 \mathrm{ml}$ is chosen for the LV. The contraction results in an end-systolic LV volume of approximately $40 \mathrm{ml}$. Both volumes are well within the reference range for healthy men [35]. The equations that govern the motion of the LV wall at every time level are derived in Appendix 1. The exact dimensions of the used LV models are included in Appendix 2.

All the geometries are meshed with approximately 800000 tetrahedral cells. The arbitrary Lagrangian-Eulerian (ALE) approach is followed, which means that the fluid grid follows the motion of the structure and subsequently needs an update to maintain good quality. This update of the fluid grid is done by remeshing and spring-based smoothing.

Blood is modelled as an incompressible Newtonian fluid with density and dynamic viscosity equal to respectively $1050 \mathrm{~kg} / \mathrm{m}^{3}$ and $4 \mathrm{E}-3 \mathrm{~Pa} \cdot \mathrm{s}$. A no-slip boundary condition is applied at the walls. No turbulence model is used, implying laminar flow.

\section{RESULTS}

The opening phase of the valve leaflets is simulated from $t=0 \mathrm{~s}$ (begin of systole) to $t=$ $0.125 \mathrm{~s}$ (peak of systole) by 250 time steps with constant time step size $(\Delta t=0.0005 \mathrm{~s})$.

\subsection{Valve leaflet kinematics}

The movement of the leaflets is depicted in Figure 3. The angular positions (Figure 3(a)) are calculated relative to the fully open position. Therefore, "0" refers to the closed position and " 1 " refers to the open leaflet.

Initially, the flow through the valve accelerates rapidly, resulting in large positive leaflet angular accelerations and increasing leaflet angular velocities. However, after this rapid flow acceleration, the acceleration of the flow through the valve starts to decrease (but remains positive until peak systole). This decrease induces negative leaflet angular accelerations and decreasing leaflet angular velocities. The peak of the leaflet angular velocity is obtained at $t=0.019 \mathrm{~s}$.

Since the resulting leaflet motion is symmetric and synchronous in the two symmetric cases (i.e. the velocity profile and the symmetric LV), only one of the two leaflets of each of these two cases is shown for clarity. Although the results for these two cases are similar, significant differences occur in the leaflet movement. In particular, the leaflet reaches the fully open position approximately $0.0015 \mathrm{~s}$ sooner when using the velocity profile. Furthermore, when using the velocity profile, the leaflet impacts the blocking mechanism with an angular velocity of $2.33 \mathrm{rad} / \mathrm{s}$, compared to an angular velocity of $1.66 \mathrm{rad} / \mathrm{s}$ in the symmetric LV case. The $40 \%$ higher angular velocity in the case with the velocity profile will result in higher stresses in the leaflets.

The above described symmetrical leaflet motion is in strong contrast to the asymmetric LV case in which a significant asymmetric and asynchronous movement of the leaflets is observed. In particular, one of the leaflets does not reach the fully open position, but achieves equilibrium at approximately 0.976 . This is in strong contrast to the other leaflet, which reaches the fully open position even sooner than in the two symmetric cases. Furthermore, this leaflet impacts the blocking mechanism at the open 
position with an angular velocity of approximately $3.12 \mathrm{rad} / \mathrm{s}$, and thus with a $34 \%$ higher velocity than when using the velocity profile.

\subsection{Valve leaflet dynamics}

Subsequently, the moments acting on the leaflets are studied (Figure 4). It is observed that the (reaction) moments acting on the leaflets in the open position (after the impact) remain the largest when using the velocity profile.

In the asymmetric LV case, the two leaflets experience different dynamics. The leaflet that reaches the fully open position (i.e. the outer leaflet), remains in this open position with a reaction moment (exerted by the blocking mechanism at the casing) that is smaller than in the case with the velocity profile, but larger than in the symmetric LV case. However, the other (inner) leaflet does not reach the open position but remains in dynamic equilibrium at position 0.976 with thus very small acting moments.

Finally, it is noted that the (peak) values of the moments at the impact depend on the temporal discretization and, therefore, a comparison of these values between the cases has no physical meaning, as is discussed in the following.

At the time of impact, the moment becomes infinite because the leaflets are considered to be stiff. However, the time integrated value of the moment (also called the impulse of the moment) has a finite value and is proportional to the sudden change in angular velocity. Since finite time steps are used in a simulation, the computed moment related to a 'sudden' velocity change will also be finite and, moreover, it will depend on the time step size. This implies that, at the time of impact, the computed value of the moment has no physical meaning (it should be infinite) and, therefore, cannot be used. This is in contrast to its time integrated value (i.e. the resulting impulse of the moment) and thus also to the sudden change in angular velocity, since both parameters are independent of the time step size for small time steps.

Therefore, in section 3.1, this sudden change in angular velocity is used (and not the value of the moment) to compare the strength of the impact between the different cases.

\subsection{Flow field}

The velocity flow field of the three cases is visualised in Figure 5 and Figure 6, respectively taken at $t=0.019 \mathrm{~s}$ (peak leaflet angular velocity) and $t=0.125 \mathrm{~s}$ (peak systole). The typical three jets downstream of the valve are clearly visible at peak systole (see Figure $6(a, b)$ and Figure 7). For the velocity profile case, the largest flow rate is observed in the middle jet at the centre of the tube. This is in contrast with the two LV cases, where very large flow rates are seen at the casing. However, in Figure 5(c) and Figure $6(c)$ it is observed that the flow upstream the valve is more uniform in the LV cases than with the velocity profile.

Furthermore, when the LV is positioned asymmetrically with respect to the valve, this results in large asymmetries in the flow field. For example, at the inflow of the valve casing in Figure $6(b, r i g h t)$ and Figure 7 , it is observed that the outer (right) jet has a larger flow rate than the inner (left) jet. Moreover, the incoming flow makes an angle with respect to the centerline of the valve casing (see Figure 8). Due to this asymmetric inflow, a large pressure is observed in Figure 8 at the inner leaflet upstream the rotation axis. 


\subsection{Spatial and temporal convergence}

The spatial and temporal convergence is studied by varying the time step size and the grid size, as is already done in [3] for the geometry with the straight tube and the velocity inlet profile.

The influence of the time step size is analysed by performing simulations with two different time step sizes: $\Delta t=0.0005 \mathrm{~s}$ and $\Delta t=0.00025 \mathrm{~s}$.

Furthermore, a spatial (grid) convergence study is performed. This is done for each of the three cases by constructing two grids with different sizes. Both grids are constructed by defining the mesh size at the leaflet walls. Subsequently, a size function is applied at these leaflet walls, which means that in the direction perpendicular to the leaflet walls, the grid size in the geometry increases at a specified rate. The first grid is the grid that is used in previous simulations, so the gap between leaflets and casing is meshed with two cell rows. Therefore, the grid measures $0.05 \mathrm{~mm}$ at the leaflet walls. It consists in total of approximately 800000 tetrahedral cells and is denoted as the "fine grid". In the second grid, one cell layer is constructed in the gap between leaflets and casing. The grid has thus size $0.1 \mathrm{~mm}$ at the leaflets and is composed of approximately 400000 tetrahedral cells. In the following, this grid is referred to as the "coarse grid".

Since the impact at the open position is a main interest in this study, the spatial and temporal convergence is checked by assessing the time level and the leaflet angular velocities of this impact. For each of the cases, three simulations are performed: the fine grid with $\Delta t=0.0005 \mathrm{~s}$, the fine grid with $\Delta t=0.00025 \mathrm{~s}$ and, finally, the coarse grid with $\Delta t=0.00025 \mathrm{~s}$. Subsequently, the time level and the mean angular velocity of the impact at the open position are calculated in each of the simulations and included in Table 1, together with their average $\mu$. It is observed that temporal and grid convergence is sufficiently achieved for the leaflet motion. The resulting leaflet movement is thus independent of the used grid resolution and the time step size.

Moreover, a comparison of the flow fields for different time step sizes and grid sizes is included. This is done by comparing the velocity fields at two time levels (i.e. $t=0.05 \mathrm{~s}$ and $t=0.1 \mathrm{~s}$ ) for the symmetric and asymmetric LV case, which are, respectively, depicted in Figure 9 and 10. The results show that for each case and at each time level, the resulting velocity fields are very similar. Therefore, they prove the temporal and grid convergence of the simulations performed in the study.

\section{DISCUSSIONS}

The results show that the leaflets impact the open position with a higher velocity in the case with the velocity profile than with the symmetrically positioned LV. Moreover, the moments on the leaflet in the open position (after the impact) remain the largest in the case with the velocity profile. Both observations are explained by the larger flow rate located at the centre of the tube when using the velocity profile (due to the development of the flow towards a parabolic shape in the upstream straight tube). The larger flow at the centre results in larger pressure and viscous forces acting at the centreline of the leaflets. Since at the centreline of the leaflet the largest leverage is obtained, these larger forces induce larger moments (with associated larger angular accelerations) in the velocity profile case, and therefore explain the above mentioned results. It can be 
reasoned that the cases with the velocity profile are less physiologically correct than the cases with the LVs, since in the LV only the flow rate is forced, whereas in the case with the velocity inlet not only the flow rate, but also the shape of the inlet profile is forced upon the flow.

Moreover, when analyzing the case with the asymmetrically positioned LV, the asymmetric inflow induces large pressures at the inner leaflet upstream of the rotation axis. This region of large pressure creates a counteracting moment that prevents the inner leaflet from opening entirely. The asymmetric flow also induces the sooner opening of the outer leaflet, since it results in a larger moment that enables the outer leaflet to reach the open position sooner and with larger velocity.

Furthermore, when analysing the impact of the leaflets at the fully open position, it is observed that one of the leaflets in the asymmetric LV case impacts the blocking mechanism with a $34 \%$ higher velocity than when using the velocity profile, and with an $88 \%$ higher velocity than in the symmetric LV case. Therefore, since a higher impact angular velocity results in higher stresses in the leaflet, this leaflet is more prone to mechanical wear and material fatigue (and thus leaflet fracture) than in the other cases.

It can be concluded that when simulating BMHVs in unphysiological (symmetrical) geometries, the mechanical stresses and the induced material fatigue (and clinically observed leaflet fracture) can be underestimated. Therefore, these results stress the need for the use of realistic boundary conditions when simulating the dynamics and structural aspects of BMHVs.

In this study, several simplifications and assumptions are made that can affect the results. Firstly, no interaction or feedback between the upstream and downstream boundary condition are taken into account.

Secondly, the model of the LV is simplified. For example, the movement of the LV wall is modelled solely by a contraction. A more physiologically correct LV model should also include the wringing of the LV wall. Moreover, the diastolic filling of the LV through the mitral valve is not taken into account and, hence, the resulting large swirling flow in the LV (as observed in $[14,16,17,18,19]$ ) is absent. Finally, the mitral valve and the aortic valve are assumed coplanar, which is physiologically not the case. It is expected that without all these simplifications the LV will yield much more asymmetry to the valve inflow, thus generating much more asymmetry in the valve leaflets dynamics. Thirdly, the valve model is simplified at the hinge regions for reasons of grid motion by cutting away the blocking mechanism and hinges at the casing. However, due to this simplification, the resulting opening angular velocity of the valve leaflets could become slightly overestimated since the additional counteracting moment created by the decelerated squeeze flows near the pivot hinge regions (i.e. the so-called pivot effect, as observed in the experiments in [36]) is absent. Nevertheless, if occurring, this pivot effect will affect the magnitude of angular velocity for all the cases in a similar way. Therefore, the relative comparison between the three different cases, as is done in this study, will remain valid.

\section{CONCLUSIONS}

When simulating the flow dynamics of (bileaflet mechanical) heart valves, the choice of the upstream boundary condition is important and impacts on the resulting leaflet motion. In particular, when using the asymmetric LV, one of the valve leaflets impacts the 
blocking mechanism at its open position with a $34 \%$ higher velocity than when using the velocity profile, and with an $88 \%$ higher velocity than in the symmetric LV case. Therefore, since a larger impact velocity results in larger stresses in the leaflet, this leaflet in the asymmetric LV will be more prone to mechanical wear and material fatigue (and thus leaflet fracture) than in the other cases. It is concluded that when simulating BMHVs in unphysiological (i.e. symmetrical) geometries, the mechanical stresses and the induced material fatigue (and clinically observed leaflet fracture) can be underestimated. Therefore, these results stress the need for the use of realistic boundary conditions when simulating the dynamics and structural aspects of such an impact.

Finally, in experimental tests, the upstream and downstream geometry is sometimes simplified into a tube with an inflow profile. Therefore, the comparison performed in this study between the velocity profile and the two more realistic inflow profiles (generated by the contracting LVs), gives a first quantification of the error that is made in such experimental tests.

\section{ACKNOWLEDGEMENTS}

Sebastiaan Annerel is funded by a BOF-grant (Special Research Fund) from Ghent University Association. Joris Degroote gratefully acknowledges a postdoctoral fellowship of the Research Foundation - Flanders (FWO).

\section{REFERENCES}

1. Butany J, Ahluwalia MS, Munroe C, Fayet C, Ahn C, Blit P, Kepron C, Cusimano RJ, Leask RL. Mechanical heart valve prostheses: identification and evaluation (erratum). Cardiovascular Pathology 2003. 12:322-344.

2. Annerel S, Degroote J, Claessens T, Vierendeels J. Evaluation of a new Implicit Coupling Algorithm for the Partitioned Fluid-Structure Interaction Simulation of Bileaflet Mechanical Heart Valves. IOP Conference Series: Materials Science and Engineering 2010. 10 : 012124.

3. Annerel S, Degroote J, Claessens T, Dahl SK, Skallerud B, Hellevik LR, Van Ransbeeck P, Segers P, Verdonck P, Vierendeels J. A Fast Strong Coupling Algorithm for the Partitioned Fluid-Structure Interaction Simulation of BMHVs. Computer Methods in Biomechanics and Biomedical Engineering 2011. In press.

4. Guivier C, Deplano V, Pibarot P. New insights into the assessment of the prosthetic valve performance in the presence of subaortic stenosis through a fluid-structure interaction model. Journal of Biomechanics 2007. $40: 2283-2290$.

5. Nobili M, Morbiducci U, Ponzini R, Del Gaudio C, Balducci A, Grigioni M, Maria Montevecchi F, Redaelli A. Numerical simulation of the dynamics of a bileaflet prosthetic heart valve using a fluid-structure interaction approaches. Journal of Biomechanics 2008. 41 : 2539-2550.

6. Dumont K, Vierendeels J, Segers P, Van Nooten G, Verdonck P. Predicting ATS Open Pivot $^{\mathrm{TM}}$ Heart Valve Performance with Computational Fluid Dynamic. Journal of Heart Valve Disease 2005. 14 : 393-399.

7. Dumont K, Vierendeels J, Kaminsky R, Van Nooten G, Verdonck P, Bluestein D. Comparison of the Hemodynamic and Thrombogenic Performance of Two Bileaflet Mechanical Heart Valves Using a CFD/FSI Model. Journal of Biomechanical Engineering 2007. $129: 558-565$. 
8. Borazjani I, Ge L, Sotiropoulos F. Curvilinear immersed boundary method for simulating fluid structure interaction with complex 3D rigid bodies. Journal of Computational Physics 2008. 227(16) : 7587-7620.

9. Borazjani I, Ge L, Sotiropoulos F. High-Resolution Fluid-Structure Interaction Simulations of Flow Through a Bi-Leaflet Mechanical Heart Valve in an Anatomic Aorta. Annals of Biomedical Engineering 2010. 38(2) : 326-344.

10. Ge L, Sotiropoulos F. A numerical method for solving the 3D unsteady incompressible Navier-Stokes equations in curvilinear domains with complex immersed boundaries. Journal of Computational Physics 2007. 225 : 1782-1809.

11. Simon HA, Ge L, Sortiropoulos F, Yoganathan A. Simulation of the Three-Dimensional Hinge Flow Fields of a Bileaflet Mechanical Heart Valve Under Aortic Conditions. Annals of Biomedical Engineering 2010. 38(3) : 841-853.

12. Guivier-Curien C, Deplano V, Bertrand E. Validation of a numerical 3-D fluid-structure interaction model for a prosthetic valve based on experimental PIV measurements. Medical Engineering \& Physics 2009. 31 : 986-993.

13. Yang J, Balaras E. An embedded-boundary formulation for large-eddy simulation of turbulent flows interacting with moving boundaries. Journal of Computational Physics 2006. $215: 12-40$.

14. Baccani B, Domenichini F, Pedrizetti G. Vortex dynamics in a model left ventricle during filling. European Journal of Mechanics B/Fluids 2002. 21 : 527-543.

15. Carmody CJ, Burriesci G, Howard IC, Patterson EA. An approach to the simulation of fluidstructure interaction in the aortic valve. Journal of Biomechanics 2006. 39 : 158-169.

16. Domenichini F, Pedrizetti G, Baccani B. Three-dimensional filling flow into a left ventricle. Journal of Fluid Mechanics 2005. 539 : 179-198.

17. Pedrizetti G, Domenichini F. Nature Optimizes the Swirling Flow in the Human Left Ventricle. Physical Review Letters 2005. 95 : 108101.

18. Querzoli G, Fortini S, Cenedese A. Effect of the prosthetic mitral valve on vortex dynamics and turbulence of the left ventricular flow. Physics of Fluids 2010. 22 : 041901.

19. Cheng Y, Oertel H, Schenkel T. Fluid-Structure Coupled CFD Simulation of the Left Ventricular Flow During Filling Phase. Annals of Biomedical Engineering 2005. 33(5) : 567576.

20. Dahl SK, Vierendeels J, Degroote J, Annerel S, Hellevik LR, Skallerud B. FSI-simulation of asymmetric mitral valve dynamics during diastolic filling. Computer Methods in Biomechanics and Biomedical Engineering 2010. DOI: 10.1080/10255842.2010.517200.

21. Doenst T, Spiegel K, Reik M, Markl M, Hennig J, Nitzsche S, Beyersdorf F, Oertel H. FluidDynamic Modeling of the Human Left Ventricle: Methodology and Application to Surgical Ventricular Reconstruction. Annals of Thoracic Surgery 2009. 97 : 1187-1195.

22. Nakamura M, Wada S, Mikami T, Kitabatake A, Karino T. Computational study on the evolution of an intraventricular vertical flow during early diastole for the interpretation of color M-mode Doppler echocardiograms. Biomechanics and Modeling in Mechanobiology 2003. $2: 59-75$.

23. Schenkel T, Malve M, Reik M, Markl M, Jung B, Oertel H. MRI-Based CFD Analysis of Flow in o Human Left Ventricle: Methodology and Application to a Healthy Heart. Annals of Biomedical Engineering 2009. 37(3) : 503-515.

24. Watanabe H, Sugiura S, Kafuku H, Hisada T. Multiphysics Simulation of Left Ventricular Filling Dynamics Using fluid-Structure Interaction Finite Element Method. Biophysical Journal 2004. 57 : 2074-2085.

25. Kaminsky R, Kallweit S, Weber H-J, Claessens T, Jozwik K, Verdonck P. Flow Visualization Through Two Types of Aortic Prosthetic Heart Valves Using Stereoscopic High-speed Particle Image Velocimetry. Artifical Organs 2007. 31(12) : 869-879. 
26. Marassi M, Castellini P, Pinotti M, Scalise L. Cardiac valve prosthesis flow performances measured by 2D and 3D-stereo particle image velocimetry. Experiments in Fluids 2004. 36 : 176-186.

27. Balducci A, Grigioni M, Querzoli G, Romano GP, Daniele C, D’Avenio G, Barbaro V. Investigation of the flow field downstream of an artificial heart valve by means of PIV and PTV. Experiments in Fluids 2004. 36 : 204-213.

28. Browne P, Ramuzat A, Saxena R, Yoganathan AP. Experimental Investigation of the Steady Flow Downstream of the St. Jude Bileaflet Heart Valve: A Comparison Between Laser Doppler Velocimetry and Particle Image Velocimetry Techniques. Annals of Biomedical Engineering 2000. 28 : 39-47.

29. Hutchison C, Sullivan P, Ethier CR. Measurements of steady flow through a bileaflet mechanical heart valve using stereoscopic PIV. Medical and Biological Engineering and Computing 2011. 49 : 325-335. DOI: 10.1007/s11517-010-0705-z

30. Vierendeels J, Dumont K, Dick E, Verdonck P. Analysis and stabilization of fluid-structure interaction algorithm for rigid-body motion. AIAA Journal 2005. 43(12) : 2549-2557.

31. Bovendeerd PHM, Arts T, Huyghe JM, van Campen DH, Reneman RS. Dependence of local left ventricular wall mechanics on myocardial fiber orientation: a model study. Journal of Biomechanics 1992. 25(10) : 1129-1140.

32. Choi HF, D'hooge J, Rademakers FE, Claus P. Influence of the left-ventricular shape on passive filling properties and end-diastolic fiber stress and strain. Journal of Biomechanics 2010. 43 : 1745-1753.

33. Rijcken J, Bovendeerd PHM, Schoofs AJG, van Campen DH, Arts T. Optimization of cardiac fiber orientation for homogeneous fiber strain at beginning of ejection. Journal of Biomechanics 1997. 30(10) : 1041-1049.

34. Vendelin M, Bovendeerd PHM, Engelbrecht J, Arts T. Optimizing ventricular fibers: uniform strain or stress, but not ATP consumption, leads to high efficiency. American Journal of Physiology - Heart and Circulatory Physiology 2002. 283(3) : H1072-H1081.

35. Lang R, Bierig M, Devereux R, Flachskampf F, Foster E, Pellikka P, Picard M, Roman M, Seward J, Shanewise J, Solomon S, Spencer K, Sutton M, Stewart W. Recommendations for chamber quantification. European Journal of Echocardiography 2006. 7 : 79-108.

36. Feng Z, Umezu M, Fujimoto T, Tsukahara T, Nurishi M, Kawaguchi D, Masuda S. Analysis of ATS leaflet behaviour by in vitro experiment. Journal of Artificial Organs 1999. 2 : 46-52. 


\section{APPENDIX 1: LV motion}

In this appendix, the equations governing the motion of the LV wall are described. The LV is modelled as a prolate truncated spheroid $[14,16,17,19,31,32,33,34]$.

\section{Equation of the surface of a truncated spheroid}

When the origin of the axes system is located at the centre of the spheroid, the surface of a spheroid (i.e. an axisymmetric ellipsoid) is generally expressed by

$$
\frac{x^{2}}{c^{2}}+\frac{y^{2}}{a^{2}}+\frac{z^{2}}{a^{2}}=1
$$

with

$$
\begin{aligned}
& -a \leq z \leq a \\
& -a \leq y \leq a \\
& -c \leq x \leq c
\end{aligned}
$$

The parameters $a$ and $c$ indicate respectively the short and the long axis of the spheroid.

In order to truncate the spheroid, the position of the intersection between a cylinder (with diameter $2 r, r \leq a$ ) and the spheroid is calculated:

$$
\left\{\begin{array}{l}
\frac{x^{2}}{c^{2}}+\frac{y^{2}}{a^{2}}+\frac{z^{2}}{a^{2}}=1 \\
y^{2}+z^{2}=r^{2}
\end{array}\right.
$$

which can be solved as

$$
x^{2}=\left(1-\frac{r^{2}}{a^{2}}\right) \cdot c^{2}
$$

resulting in

$$
x= \pm c \sqrt{1-\frac{r^{2}}{a^{2}}}
$$

Therefore, the equation that describes the surface of a truncated spheroid is given by

$$
\frac{x^{2}}{c^{2}}+\frac{y^{2}}{a^{2}}+\frac{z^{2}}{a^{2}}=1
$$

with

$$
\begin{gathered}
-a \leq z \leq a \\
-a \leq y \leq a \\
-c \sqrt{1-\frac{r^{2}}{a^{2}}} \leq x \leq c
\end{gathered}
$$

The volume of the $L V$

The volume of the truncated spheroid is calculated as: 


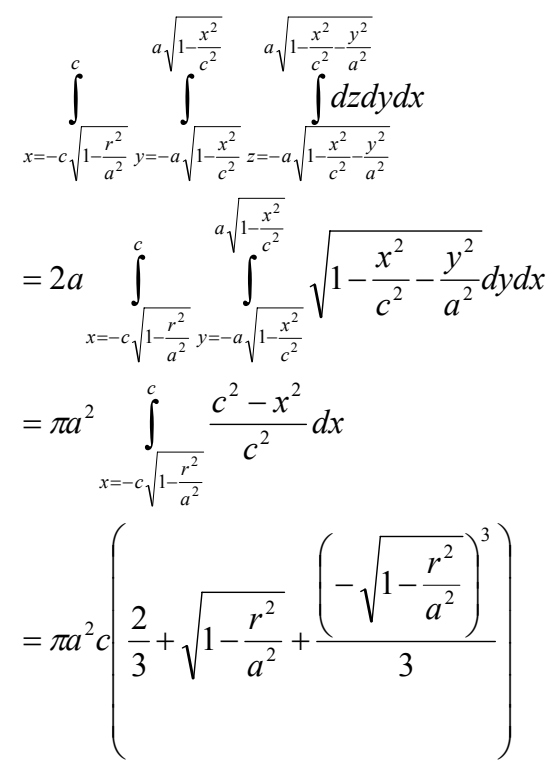

The parameters $a$ and $c$, that determine the shape of the spheroid, are a function of the time. Therefore, at every time level $t$, the volume $V(t)$ is given by:

$$
V(t)=\pi \cdot a(t)^{2} \cdot c(t)\left(\frac{2}{3}+\sqrt{1-\frac{r^{2}}{a(t)^{2}}}+\frac{\left(-\sqrt{1-\frac{r^{2}}{a(t)^{2}}}\right)^{3}}{3}\right)
$$

Moreover, the coefficient $a(t)$ is set as a constant relation with $c(t)$ :

$$
a(t)=\frac{c(t)}{\alpha}
$$

By doing this, the spheroid is controlled by one parameter $c(t)$, since the parameter $\alpha$ is usually fixed in case of a LV. When $\alpha>1$, the spheroid is called "prolate".

Equation (9) becomes:

$$
V(t)=\pi \cdot \frac{c(t)^{3}}{\alpha^{2}}\left(\frac{2}{3}+\sqrt{1-\frac{\alpha^{2} \cdot r^{2}}{c(t)^{2}}}+\frac{\left(-\sqrt{1-\frac{\alpha^{2} \cdot r^{2}}{c(t)^{2}}}\right)^{3}}{3}\right)
$$

\section{Calculation of the value $c(t+\Delta t)$ :}

When using the velocity profile, a flow rate $Q(t+\Delta t)$ flows into the straight tube (with diameter $r$ ) at each time level $t+\Delta t$ :

$$
Q(t+\Delta t)=v(t+\Delta t) \cdot \pi \cdot r^{2}
$$

with $v(t+\Delta t)$ the aortic flow velocity at time level $t+\Delta t$, as visualized in Figure 3(a). This flow rate can also be obtained by a contraction of the truncated spheroid:

$$
V(t+\Delta t)=V(t)+Q(t+\Delta t) \cdot \Delta t
$$

From Equation (12) and (13) the appropriate value for $c(t+\Delta t)$ is calculated at every time level $t+\Delta t$ by Newton iterations.

\section{Calculation of the movement of the $L V$ wall at time level $t+\Delta t$ :}

The diameter and (absolute) coordinates of the truncation is fixed in the simulations. Therefore, the spheroid will not only reduce in volume during the contraction, but it will also undergo a translation in 
order to maintain a correct truncation. Therefore, in order to simplify the wall motion, the axes system is translated from the centre of the spheroid to the centre of the truncation. The equation describing the position of the LV wall at time level $t$ is thus given by:

$$
\frac{\left(x(t)-c(t) \sqrt{1-\frac{\alpha^{2} \cdot r^{2}}{c(t)^{2}}}\right)^{2}}{c(t)^{2}}+\frac{\alpha^{2} \cdot z(t)^{2}}{c(t)^{2}}+\frac{\alpha^{2} \cdot y(t)^{2}}{c(t)^{2}}=1
$$

Moreover, no wringing of the LV wall is allowed, only contraction. Therefore, the azimuth $(\pi / 2-\varphi)$ and the inclination $\theta$ of each point at the $\mathrm{LV}$ wall is constant:

$$
\begin{aligned}
& \varphi=C s t \Leftrightarrow \tan \varphi=\frac{\sqrt{y^{2}+z^{2}}}{x}=C t e \\
& \theta=C s t \Leftrightarrow \tan \theta=\frac{y}{z}=C t e
\end{aligned}
$$

The values for $\tan \theta$ and $\tan \varphi$ can be calculated from the coordinates $(x(t), y(t), z(t))$ at time level $t$ :

$$
\left\{\begin{array}{l}
\tan (\varphi(t))=\frac{\sqrt{y(t)^{2}+z(t)^{2}}}{x(t)} \\
\tan (\theta(t))=\frac{y(t)}{z(t)}
\end{array}\right.
$$

And, thus, at time level $t+\Delta t$ :

$$
\left\{\begin{array}{l}
\tan (\varphi(t+\Delta t))=\frac{\sqrt{y(t+\Delta t)^{2}+z(t+\Delta t)^{2}}}{x(t+\Delta t)}=\tan (\varphi(t)) \\
\tan (\theta(t+\Delta t))=\frac{y(t+\Delta t)}{z(t+\Delta t)}=\tan (\theta(t))
\end{array}\right.
$$

Moreover, the equation for the spheroid's surface at this new time level is:

$$
\frac{\left(x(t+\Delta t)-c(t+\Delta t) \sqrt{1-\frac{\alpha^{2} \cdot r^{2}}{c(t+\Delta t)^{2}}}\right)^{2}}{c(t+\Delta t)^{2}}+\frac{\alpha^{2} \cdot z(t+\Delta t)^{2}}{c(t+\Delta t)^{2}}+\frac{\alpha^{2} \cdot y(t+\Delta t)^{2}}{c(t+\Delta t)^{2}}=1
$$

Since

$$
y(t+\Delta t)^{2}+z(t+\Delta t)^{2}=\tan (\varphi(t))^{2} \cdot x(t+\Delta t)^{2}
$$

the previous equation can be rewritten as

$$
\frac{\left(x(t+\Delta t)-c(t+\Delta t) \sqrt{1-\frac{\alpha^{2} \cdot r^{2}}{c(t+\Delta t)^{2}}}\right)^{2}}{c(t+\Delta t)^{2}}+\frac{\alpha^{2} \cdot \tan (\varphi(t))^{2} \cdot x(t+\Delta t)^{2}}{c(t+\Delta t)^{2}}=1
$$

and solved for $x(t+\Delta t)$, giving:

$$
x(t+\Delta t)=\frac{\sqrt{(c(t+\Delta t)-\alpha \cdot r) \cdot(c(t+\Delta t)+\alpha \cdot r)} \pm \sqrt{c(t+\Delta t)^{2}+\alpha^{4} \cdot r^{2} \cdot \tan (\varphi(t))^{2}}}{\alpha^{2} \cdot \tan (\varphi(t))^{2}+1}
$$

Previous equation is only used with "+", because "-" lies in the part of the spheroid that is cut off by the truncation.

From

$$
\left\{\begin{array}{l}
y(t+\Delta t)^{2}+z(t+\Delta t)^{2}=\tan (\varphi(t))^{2} \cdot x(t+\Delta t)^{2} \\
y(t+\Delta t)=\tan (\theta(t)) \cdot z(t+\Delta t)
\end{array}\right.
$$

the new coordinates for $y(t+\Delta t)$ and $z(t+\Delta t)$ are solved

$$
\left\{\begin{array}{l}
z(t+\Delta t)^{2}=\frac{\tan (\varphi(t))^{2} \cdot x(t+\Delta t)^{2}}{\left(1+\tan (\theta(t))^{2}\right)} \\
y(t+\Delta t)=\tan (\theta(t)) \cdot z(t+\Delta t)
\end{array}\right.
$$


In summary, the new coordinates $(x(t+\Delta t), y(t+\Delta t), z(t+\Delta t))$ of each point at the LV wall are calculated as a function of the coordinates at previous time level $t$ :

$$
\left\{\begin{array}{l}
x(t+\Delta t)=\frac{\sqrt{(c(t+\Delta t)-\alpha \cdot r) \cdot(c(t+\Delta t)+\alpha \cdot r)}+\sqrt{c(t+\Delta t)^{2}+\alpha^{4} \cdot r^{2} \cdot \tan (\varphi(t))^{2}}}{\alpha^{2} \cdot \tan (\varphi(t))^{2}+1} \\
z(t+\Delta t)^{2}=\frac{\tan (\varphi(t))^{2} \cdot x(t+\Delta t)^{2}}{\left(1+\tan (\theta(t))^{2}\right)} \\
y(t+\Delta t)=\tan (\theta(t)) \cdot z(t+\Delta t)
\end{array}\right.
$$

\section{APPENDIX 2: Dimensions of the used LV models}

In this appendix, the dimensions of the end-diastolic and end-systolic volumes of the used LV models are summarized. All the dimensions of the volumes and inner diameters are consistent with the reference range for normal healthy men in [35]. Since the long-to-short-axis ratio is kept fixed at 2, the value for $\alpha$ is equal to 2 [32].

First, the dimensions of the end-diastolic LV volumes (of $111 \mathrm{ml}$ ) are given:

For the symmetrically positioned LV:

$$
\begin{aligned}
c & =47.53 \mathrm{~mm} ; \\
r & =11.09 \mathrm{~mm} .
\end{aligned}
$$

For the asymmetrically positioned LV:

$$
\begin{aligned}
& c=48.52 \mathrm{~mm} ; \\
& r=18 \mathrm{~mm} .
\end{aligned}
$$

After the contraction, the following end-systolic LV dimensions (volume $=40 \mathrm{ml}$ ) are obtained:

For the symmetrically positioned LV:

$$
c=34.14 \mathrm{~mm}
$$$$
r=11.09 \mathrm{~mm} \text {. }
$$

For the asymmetrically positioned LV:

$c=37.66 \mathrm{~mm}$;

$r=18 \mathrm{~mm}$. 


\section{LIST OF TABLES AND FIGURES}

Table 1: Sensitivity to the temporal and spatial resolution of the calculated time level and leaflet angular velocity when impacting the open position. For each case, the average $\mu$ is calculated.

Figure 1: View on the ATS Open Pivot ${ }^{\mathrm{TM}}$ Standard Heart Valve with leaflets marked in black in an opened position. The casing is visible (in white). (a) without simplification of the blocking mechanism at the hinges; $(b)$ with simplification of the blocking mechanism and the hinges.

Figure 2: View on the geometry of the simulated cases, with the boundary conditions. Downstream: pressure outlet (in red). Upstream: (a) inflow velocity profile (in blue), $(b)$ symmetrically positioned contracting $\mathrm{LV},(c)$ asymmetrically positioned contracting LV.

Figure 3: Plot of the aortic flow velocity $(a)$ and the leaflet movement: angular position $(a)$, angular velocity $(b)$ and angular acceleration $(c)$. Aortic flow velocity $(\cdots)$, Leaflet with velocity profile (-), Leaflet with symmetrically positioned LV (---), Inner leaflet with asymmetrically positioned LV (--), Outer leaflet with asymmetrically positioned LV (-・-). The impact at the open position is shown magnified at the right.

Figure 4: Plot of the moments. Leaflet with velocity profile (-), Leaflet with symmetrically positioned LV (---), Inner leaflet with asymmetrically positioned LV (---), Outer leaflet with asymmetrically positioned LV (-.-). The impact at the open position is shown magnified at the right.

Figure 5: Velocity flow field (in $\mathrm{m} / \mathrm{s}$ ) during opening $(t=0.019 \mathrm{~s})$. (left $)$ inflow velocity profile, (middle) symmetric LV, (right) asymmetric LV. (a) longitudinal section perpendicular to leaflet rotation axes, $(b)$ cross-section at the inflow of the valve casing, $(c)$ axial section between the two leaflets.

Figure 6: Velocity flow field (in $\mathrm{m} / \mathrm{s})$ at peak systole $(t=0.125 \mathrm{~s})$. (left $)$ inflow velocity profile, (middle) symmetric LV, (right) asymmetric LV. (a) longitudinal section perpendicular to leaflet rotation axes, $(b)$ cross-section at the inflow of the valve casing, (c) axial section between the two leaflets.

Figure 7: Plot of the flow velocity magnitude (in $\mathrm{m} / \mathrm{s}$ ) as function of the transversal position at peak systole $(t=0.125 \mathrm{~s})$. Shown is the intersection between the longitudinal section (Figure 5(a) $\& 6(a)$ ) and the inflow section (Figure $5(b) \& 6(b))$ for each of the cases.

Figure 8: View on the asymmetrically positioned LV case at peak systole $(t=0.125 \mathrm{~s})$. Velocity vectors are shown in black on the longitudinal section, as already used in Figure 5(a) and Figure 6(a). The pressure levels (in Pa) are coloured on the leaflets surfaces, clearly indicating the high pressure region at the inner leaflet upstream the hinge axis.

Figure 9: Sensitivity study of the velocity field (in $\mathrm{m} / \mathrm{s}$ ) to the spatial and temporal discretization for the symmetric LV case: (up) $t=0.05 \mathrm{~s} ;($ down $) t=0.1 \mathrm{~s}$; (a) fine grid and $\Delta t=0.0005 \mathrm{~s}$; $(b)$ fine grid and $\Delta t=0.00025 \mathrm{~s} ;(c)$ coarse grid and $\Delta t=0.00025 \mathrm{~s}$.

Figure 10: Sensitivity study of the velocity field (in $\mathrm{m} / \mathrm{s}$ ) to the spatial and temporal discretization for the asymmetric LV case: $(u p) t=0.05 \mathrm{~s} ;($ down $) t=0.1 \mathrm{~s} ;(a)$ fine grid and $\Delta t=0.0005 \mathrm{~s} ;(b)$ fine grid and $\Delta t=0.00025 \mathrm{~s} ;(c)$ coarse grid and $\Delta t=0.00025 \mathrm{~s}$. 


\begin{tabular}{c|c|c|c|c|c|c|c}
\multicolumn{2}{l|}{} & \multicolumn{2}{c|}{ Velocity profile } & \multicolumn{2}{c|}{ Symmetric LV } & \multicolumn{2}{c}{$\begin{array}{c}\text { Asymmetric LV } \\
\text { Outer leaflet }\end{array}$} \\
\hline \multicolumn{2}{l|}{} & $t$ (in s) & $\begin{array}{c}\text { Velocity } \\
\text { (in rad/s) }\end{array}$ & $t$ (in s) & $\begin{array}{c}\text { Velocity } \\
\text { (in rad/s) }\end{array}$ & $t$ (in s) & $\begin{array}{c}\text { Velocity } \\
\text { (in rad/s) }\end{array}$ \\
\hline Coarse & $\boldsymbol{\Delta} \boldsymbol{t}=\mathbf{0 . 0 0 0 2 5 s}$ & 0.06825 & 2.284 & 0.06925 & 1.403 & 0.06575 & 2.653 \\
\hline Fine & $\boldsymbol{\Delta} \boldsymbol{t}=\mathbf{0 . 0 0 0 2 5 s}$ & 0.06725 & 2.475 & 0.06850 & 1.650 & 0.06425 & 2.728 \\
Fine & $\boldsymbol{\Delta} \boldsymbol{t}=\mathbf{0 . 0 0 0 5 0 s}$ & 0.06850 & 2.332 & 0.07000 & 1.658 & 0.06350 & 3.123 \\
\hline $\boldsymbol{\mu}$ & & 0.06800 & 2.364 & 0.06925 & 1.570 & 0.06450 & 2.835
\end{tabular}

Table 1: Sensitivity to the temporal and spatial resolution of the calculated time level and leaflet angular velocity when impacting the open position. For each case, the average $\mu$ is calculated. 


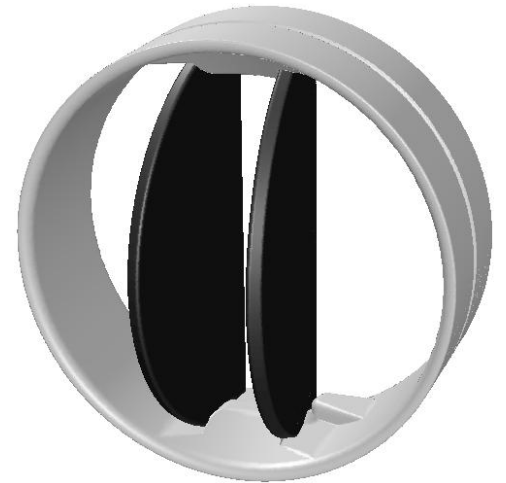

(a)

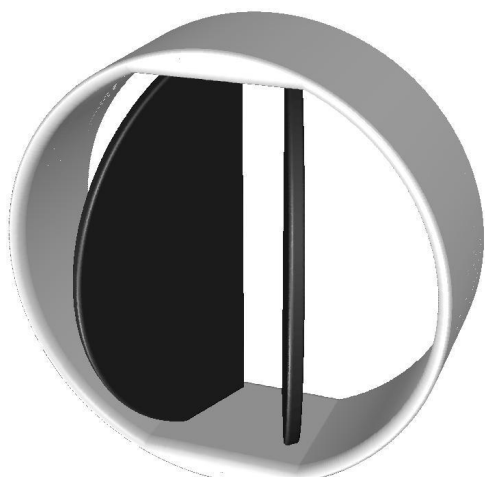

(b)

Figure 1: View on the ATS Open Pivot ${ }^{\mathrm{TM}}$ Standard Heart Valve with leaflets marked in black in an opened position. The casing is visible in white. $(a)$ without simplification of the blocking mechanism at the hinges; $(b)$ with simplification of the blocking mechanism and the hinges. 


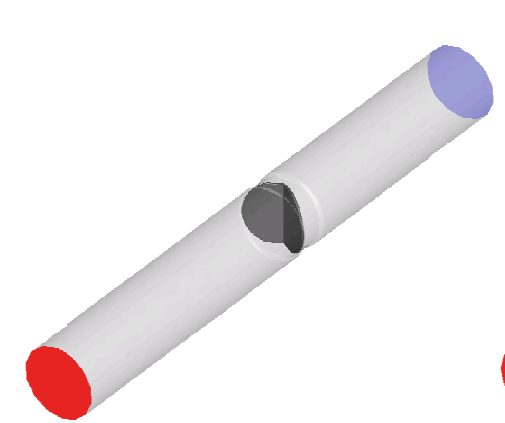

(a)

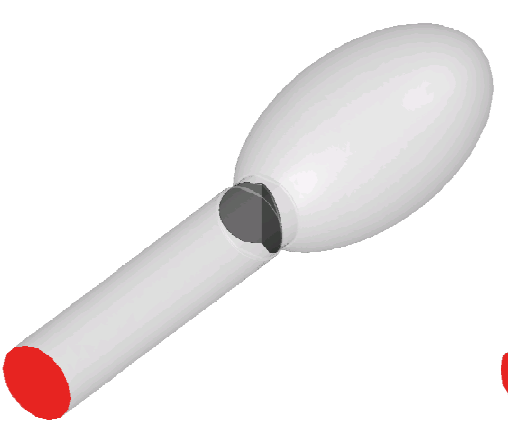

(b)

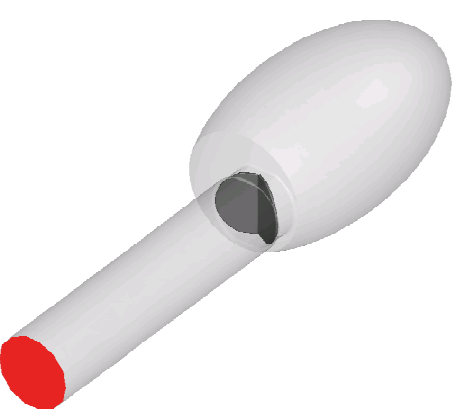

(c)

Figure 2: View on the geometry of the simulated cases, with the boundary conditions. Downstream: pressure outlet (in red). Upstream: $(a)$ inflow velocity profile (in blue), $(b)$ symmetrically positioned contracting LV, (c) asymmetrically positioned contracting LV. 
(a)
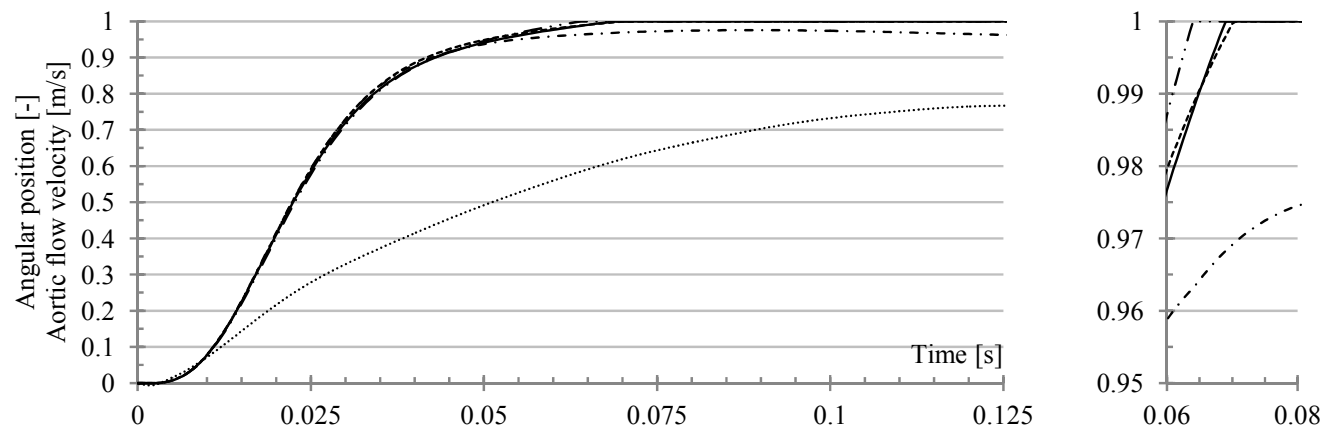

(b)
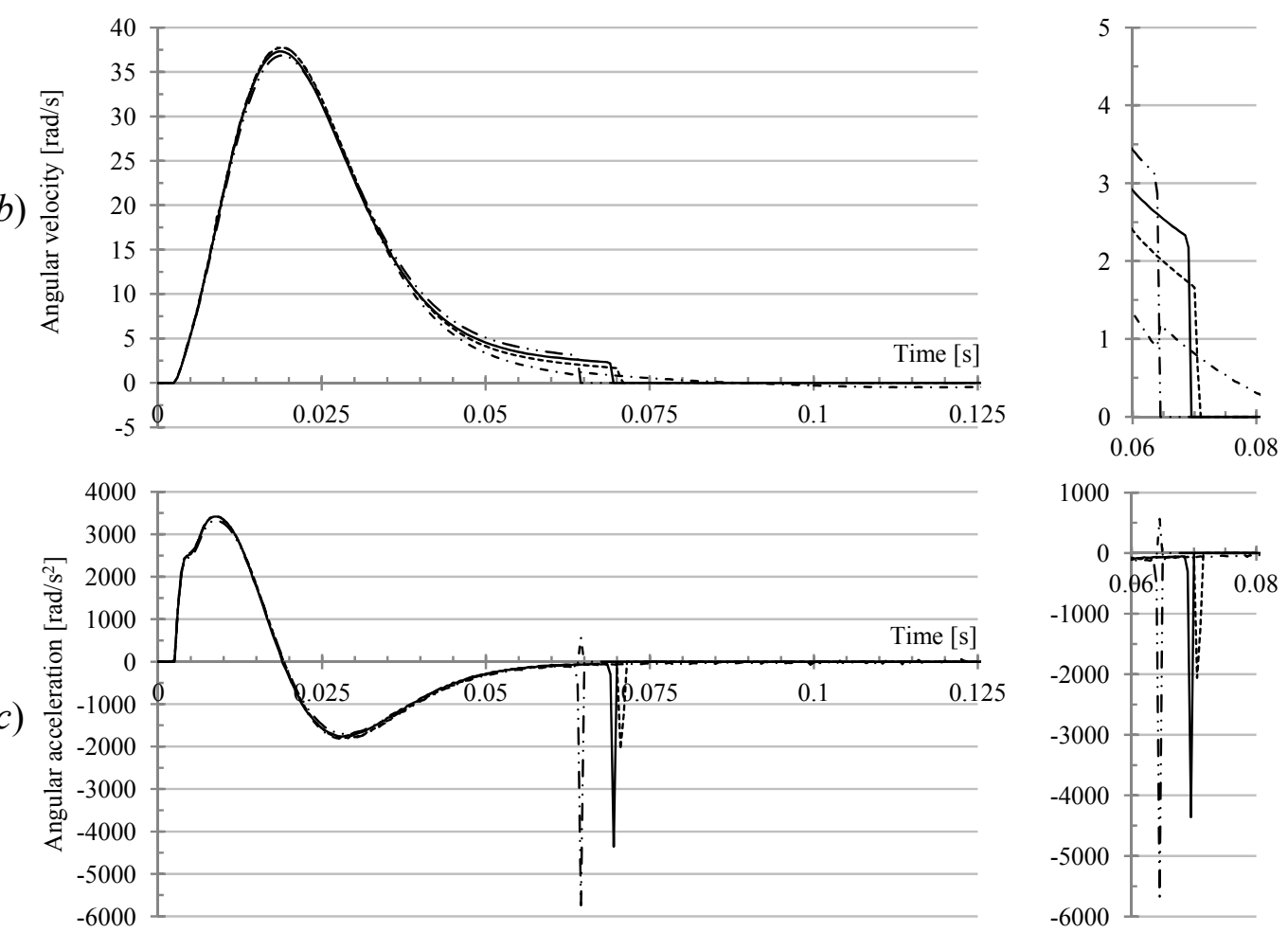

Figure 3: Plot of the aortic flow velocity $(a)$ and the leaflet movement: angular position $(a)$, angular velocity $(b)$ and angular acceleration $(c)$. Aortic flow velocity $(\cdots)$, Leaflet with velocity profile (-), Leaflet with symmetrically positioned LV (---), Inner leaflet with asymmetrically positioned LV (-•-), Outer leaflet with asymmetrically positioned LV $(-\cdots)$. The impact at the open position is shown magnified at the right. 

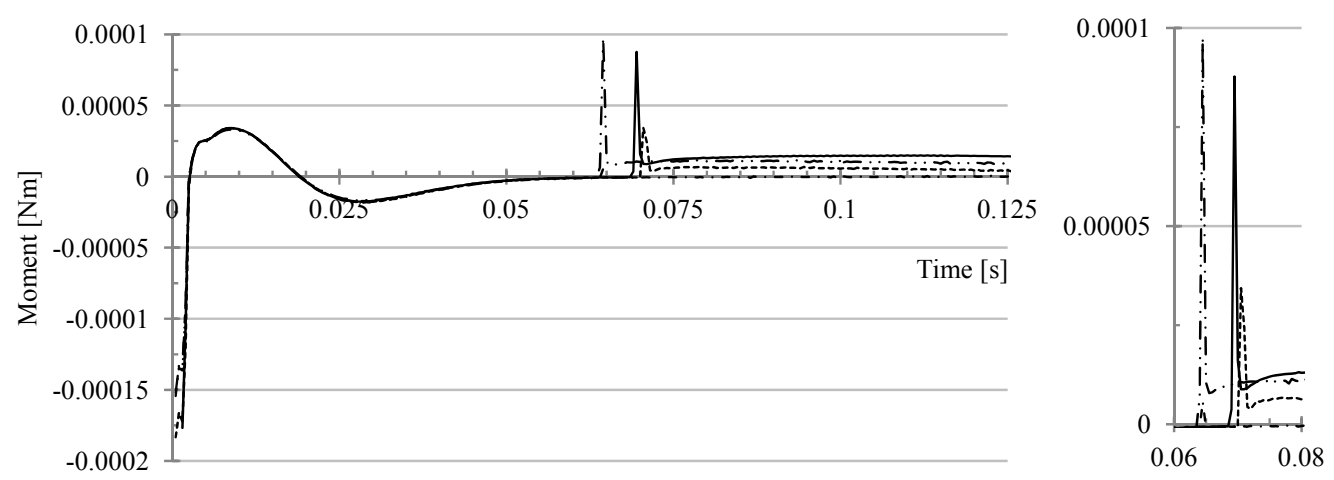

Figure 4: Plot of the moments. Leaflet with velocity profile (-), Leaflet with symmetrically positioned LV (---), Inner leaflet with asymmetrically positioned LV (---), Outer leaflet with asymmetrically positioned LV (-.--). The impact at the open position is shown magnified at the right. 

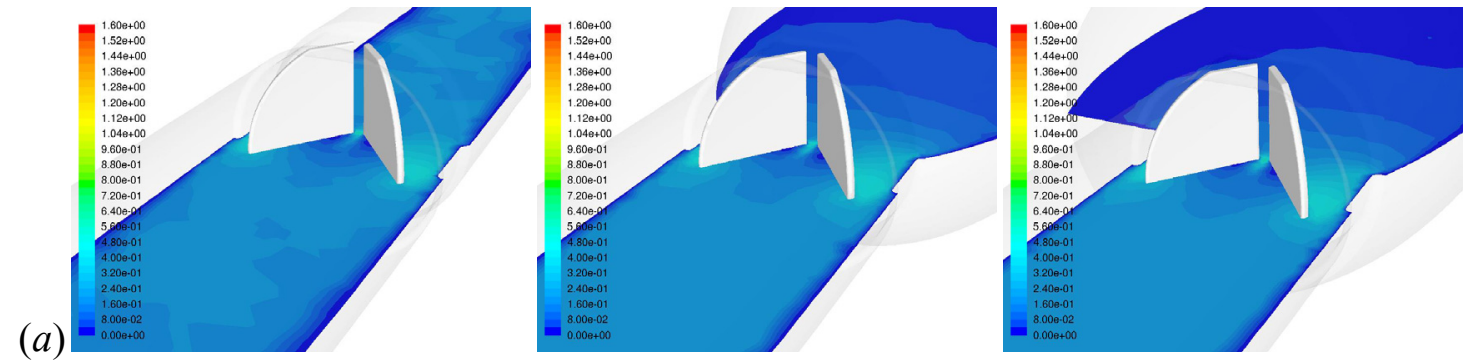

(b)
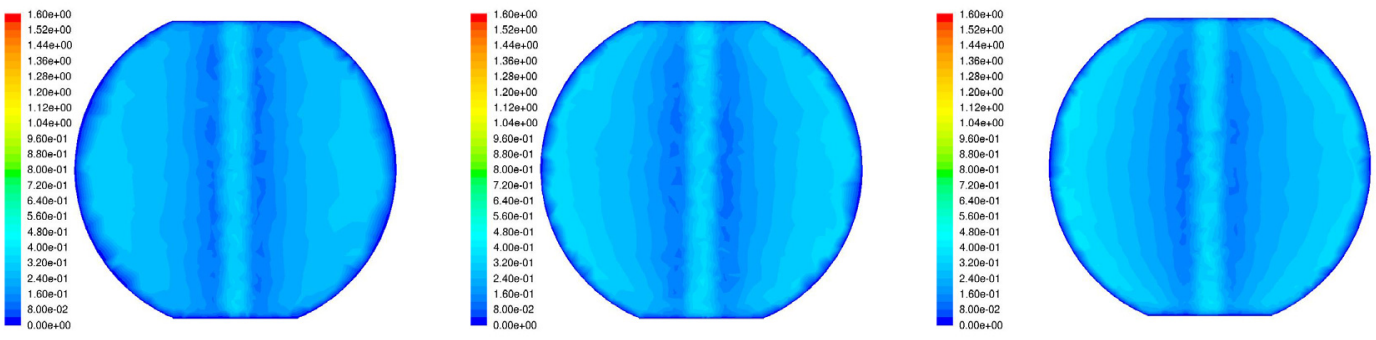

(c)
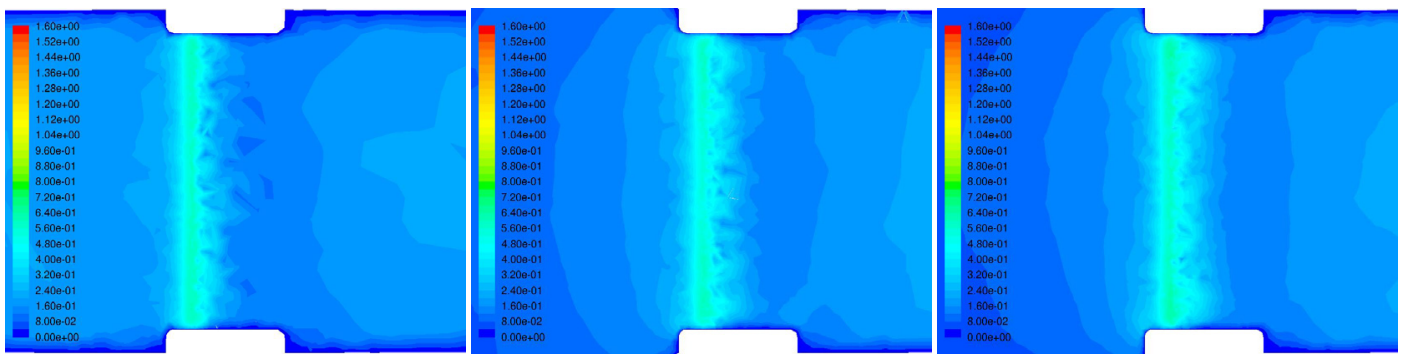

Figure 5: Velocity flow field (in $\mathrm{m} / \mathrm{s})$ during opening $(t=0.019 \mathrm{~s})$. (left) inflow velocity profile, (middle) symmetric LV, (right) asymmetric LV. (a) longitudinal section perpendicular to leaflet rotation axes, $(b)$ cross-section at the inflow of the valve casing, (c) axial section between the two leaflets. 


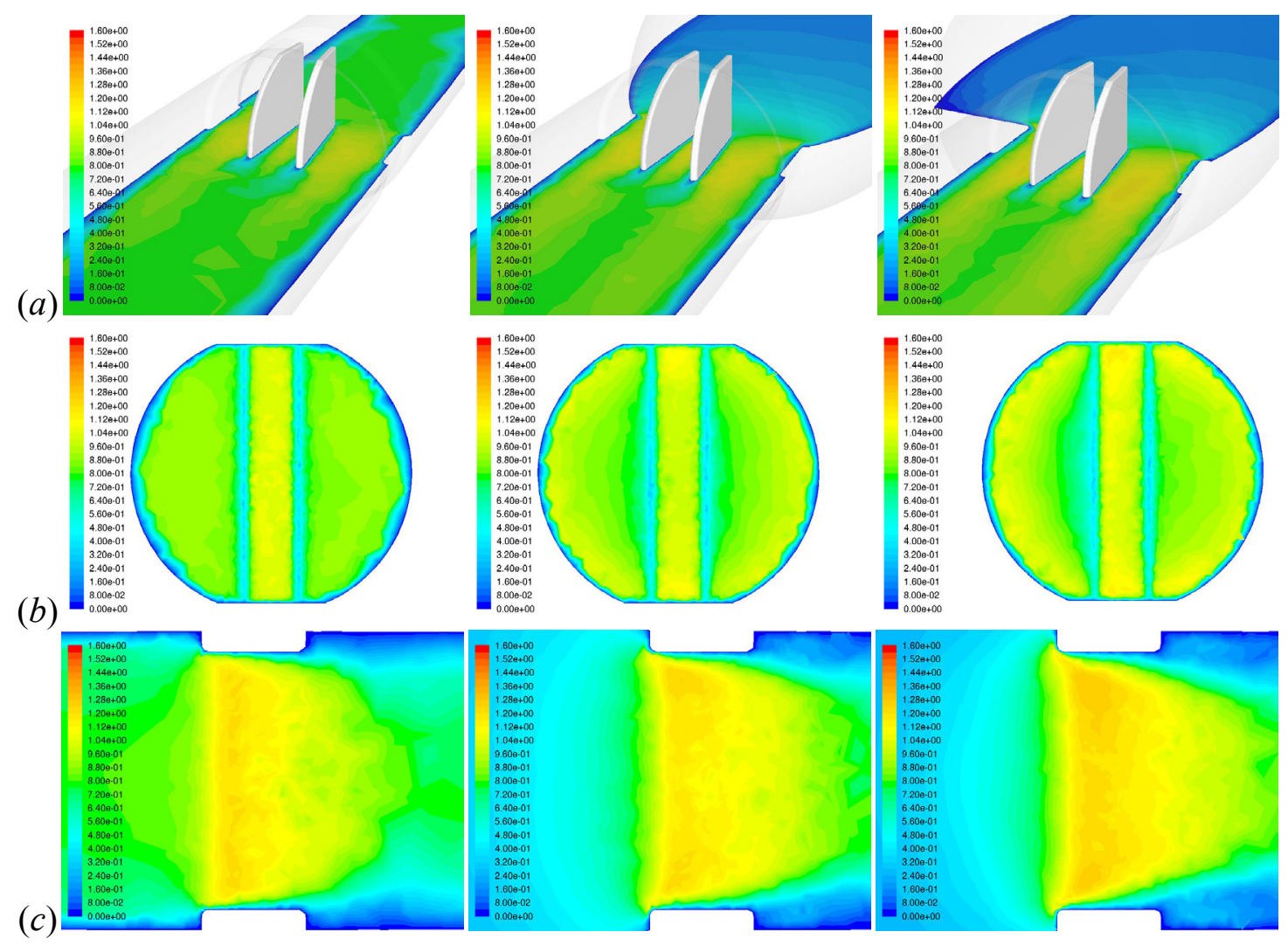

Figure 6: Velocity flow field (in $\mathrm{m} / \mathrm{s})$ at peak systole $(t=0.125 \mathrm{~s})$. (left) inflow velocity profile, (middle) symmetric LV, (right) asymmetric LV. (a) longitudinal section perpendicular to leaflet rotation axes, $(b)$ cross-section at the inflow of the valve casing, (c) axial section between the two leaflets. 


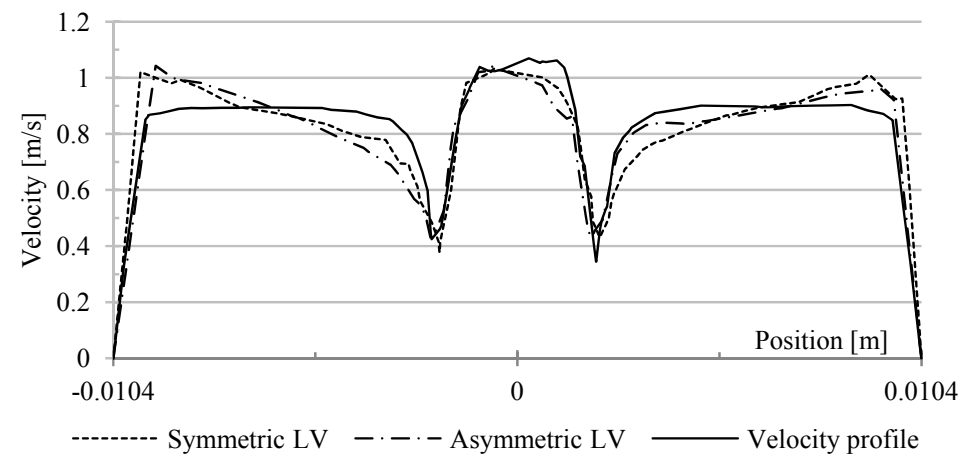

Figure 7: Plot of the flow velocity magnitude (in $\mathrm{m} / \mathrm{s}$ ) as function of the transversal position at peak systole $(t=0.125 \mathrm{~s})$. Shown is the intersection between the longitudinal section (Figure 5(a) \& 6(a)) and the inflow section (Figure 5(b) \& 6(b)) for each of the cases. 


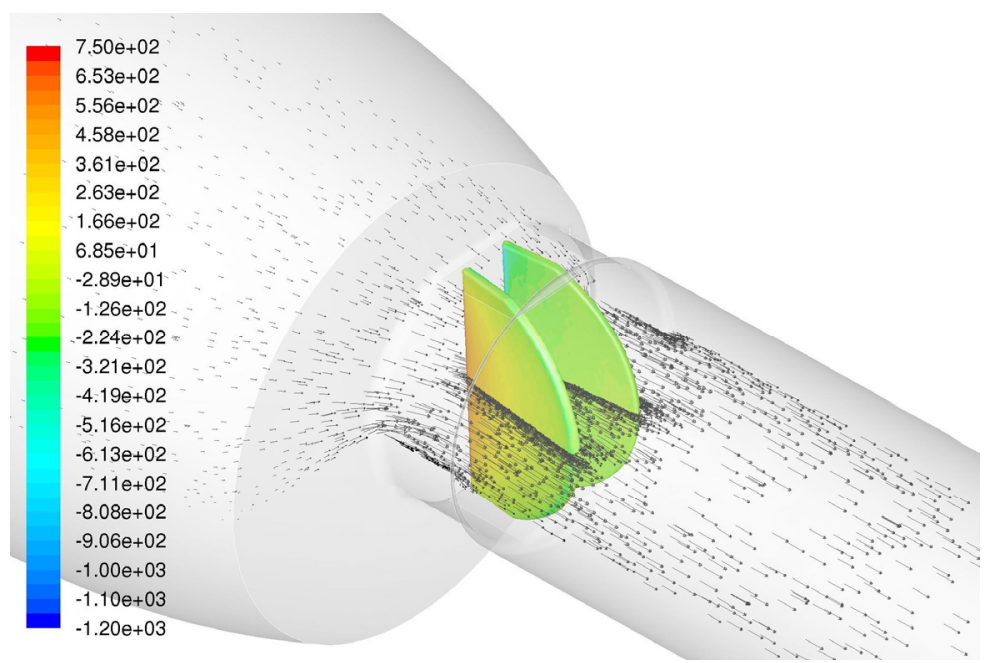

Figure 8: View on the asymmetrically positioned LV case at peak systole $(t=0.125 \mathrm{~s})$. Velocity vectors are shown in black on the longitudinal section, as already used in Figure 5(a) and Figure 6(a). The pressure levels (in Pa) are coloured on the leaflet surfaces, clearly indicating the high pressure region at the inner leaflet upstream the hinge axis. 

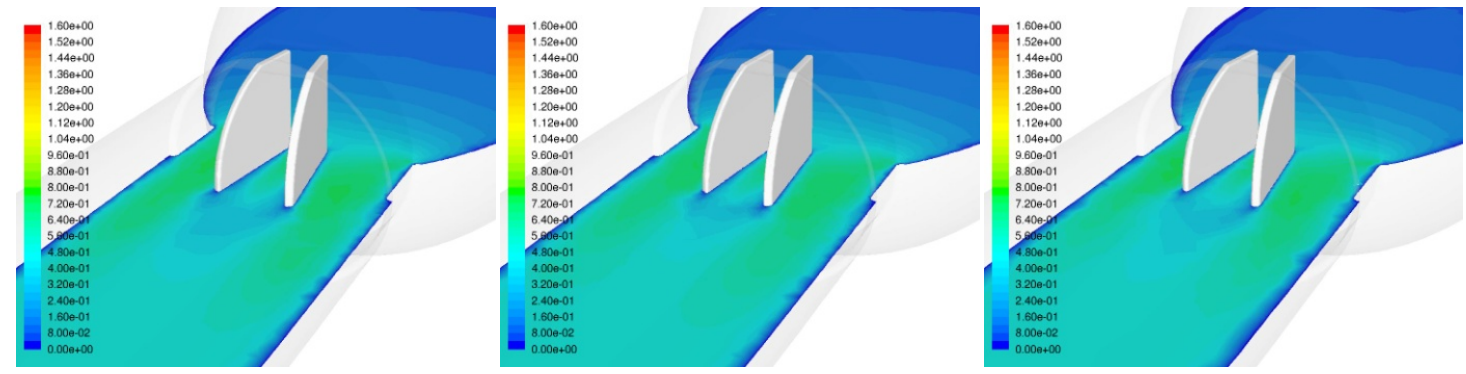

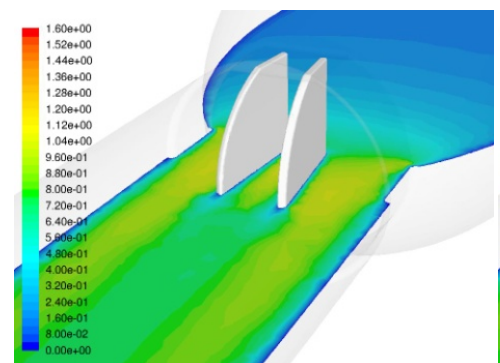

(a)

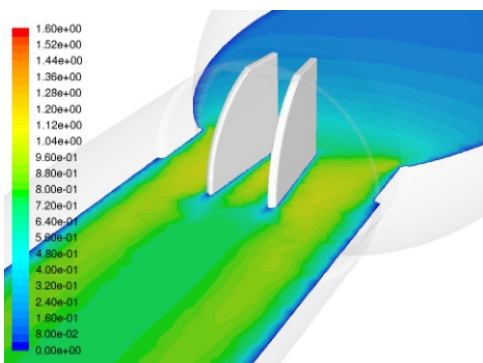

(b)

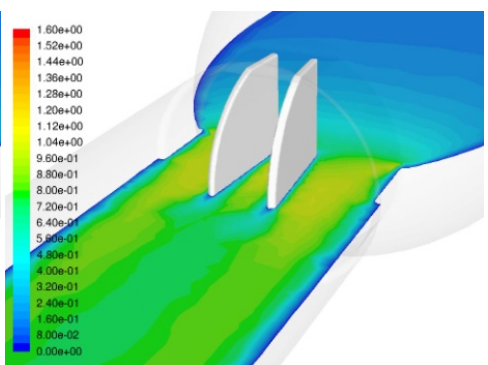

(c)

Figure 9: Sensitivity study of the velocity field (in $\mathrm{m} / \mathrm{s}$ ) to the spatial and temporal discretization for the symmetric LV case: $(u p) t=0.05 \mathrm{~s} ;($ down $) t=0.1 \mathrm{~s} ;(a)$ fine grid and $\Delta t=0.0005 \mathrm{~s} ;(b)$ fine grid and $\Delta t=0.00025 \mathrm{~s} ;(c)$ coarse grid and $\Delta t=0.00025 \mathrm{~s}$. 

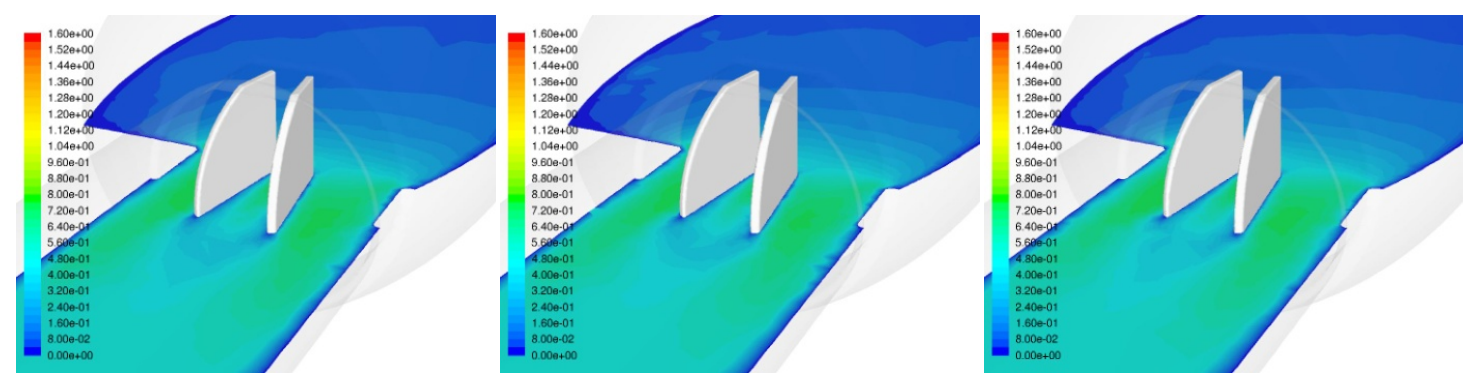

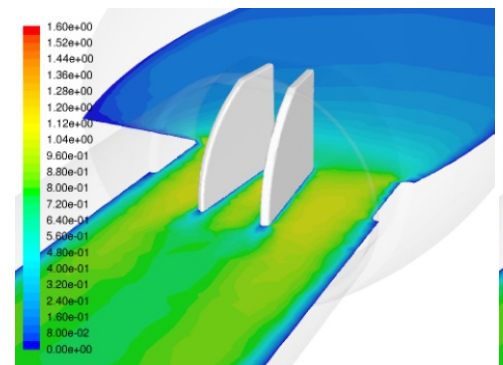

(a)

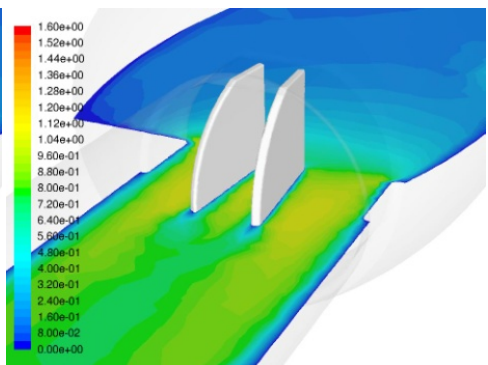

(b)

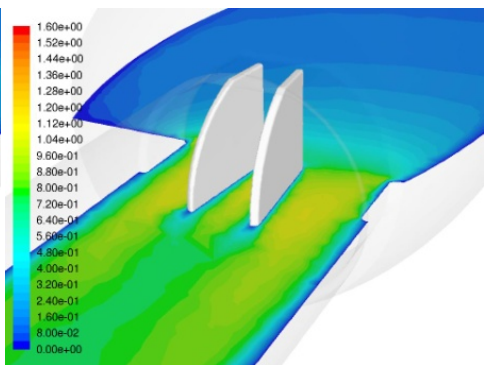

(c)

Figure 10: Sensitivity study of the velocity field (in $\mathrm{m} / \mathrm{s}$ ) to the spatial and temporal discretization for the asymmetric LV case: (up) $t=0.05 \mathrm{~s}$; (down) $t=0.1 \mathrm{~s}$; (a) fine grid and $\Delta t=0.0005 \mathrm{~s} ;(b)$ fine grid and $\Delta t=0.00025 \mathrm{~s} ;(c)$ coarse grid and $\Delta t=0.00025 \mathrm{~s}$. 